\section{HEREDITARY TYROSINEMIA TYPE I: LIVER TRANSPLANTAION (LT) WITH LIVING DONOR FOR HEPATOCARCINOMA AFTER 7 YEARS OF NTBC}

\author{
E. Barkaoui' ${ }^{1}$ S. Mannai ${ }^{2}$, H. Mestiri², \\ K. Boujemaa ${ }^{3}$, S. Abbès ${ }^{1}$
}

${ }^{1}$ Pasteur Institut, Tunis, ${ }^{2}$ Surgery Center, Marsa, Tunisia, ${ }^{3}$ Surgery Center, Rennes, France

Backgrounand aim: Hereditary tyrosinemia type I $(\mathrm{HTI})$ is a severe metabolic disease due to deficiency of fumaryl aceto acetate hydrolase (FAAH). The main clinical consequence of this defect includes hepatic involvement, high risque of liver cancer, renal tubular dysfunction and neurological crisis. Until 1992 transplantation is the only treatment of HTI. Since 1992, the NTBC (2 - (2 - nitro-4 triofluoromethyl benzoyl) - 1.3 - cyclohexane dione) offers a new promising tool for the treatment of HTI. We report the results of this treatment in one Tunisian patients who developped hepatocarcinoma after 7.5 years of treatemnt.

Observation: The disease was revealed by subacute form at age 9 months by hepatic dysfunction. The diagnosis was confirmed by urinary abnormal excretion of succinyl acetone (SA) and $\Delta$ amino levulinate. The treatment by NTBC was started $r$ at age of 10 months. The hepatic function was normalized at one monthAfter 7 years, he developed an hepatocarcinoma with reascention of alpha foetoproteine. In the first time the child have a resection of tumor than a liver transplantation with living donor and now he is at 4 years anf 8 months of the LT. te rate of the suuccinyl acetone was rased at $21 \mu \mathrm{mol} / \mathrm{l}$.

Conclusion: The NTBC is a promising alternative treatment of $\mathrm{HTI}$ to the transplantation and the results reported here highlight the efficiency of the NTBC in early outcome however it doesn't prevent at the long term from the liver cancer.

\section{5}

\section{ETIOLOGY OF UPPER GASTROINTESTINAL BLEEDING IN CHILDREN}

\author{
L. Mihai ${ }^{1}$, A. Balasa ${ }^{1}$, C.M. Mihai ${ }^{1}$, V. Stroia ${ }^{1}$, \\ V. Cuzic ${ }^{1}$, C. Frecus ${ }^{1}$, R. Stoicescu ${ }^{2}$ \\ ${ }^{1}$ Pediatric, ${ }^{2}$ Microbiology, Ovidius University, \\ Constanta, Romania
}

Background: Upper gastrointestinal bleeding (UGIB) is a relatively common and occasionally fatal emergency in children. Upper digestive endoscopy is the method of choice when establish the etiology.

Objective: to determine the etiology of UGIB, indications for upper digestive endoscopy, the safety of the procedure in children.

Material and method: 129 children admitted in Pediatric Department, Clinical Hospital Constanta, Romania, with UGIB, undergone a number of 178 upper digestive endoscopies in a prospective study during 5 years. Endoscopy was performed under local/iv general anesthesia/iv sedation, depending on age and agreement of children and parents, after the procedure was explained.

Results: 66 boys and 63 girls aged between 9 months and 16 years (mean age 9.7 years) undergone upper digestive endoscopy for hematemesis (95 cases), melena (88 cases) and inexplicable anemia (4 cases). Endoscopy detected the site of bleeding in 118 cases $(91.47 \%)$. Association of hematemesis and melena was best correlated with endoscopical findings $(96.55 \%)$. The etiology revealed most frequently erosive gastritis (59 cases), esophageal varices (24 cases), duodenal ulcers (10 cases), erosive esophagitis (10 cases), erosive duodenitis (8 cases). We found rare etiology in children, such as gastric ulcer (2 cases), duodenal angiodisplasia (2 cases), gastric polyp (1 case), Mallory Weiss syndrome (1 case), gastric adenocarcinoma (1 case). We used local anesthesia (46 cases), iv anesthesia (62 cases) and iv sedation (70 cases). No complications were encountered.

Conclusion: Upper digestive endoscopy, was a safe procedure in children, visualizing recent stigmas of hemorrhage in $91,47 \%$ of cases of UGIB.

\section{6}

\section{ANTIBODIES OF HEPATITIS A IN CHILDREN IN SYRIA}

\section{Anjak \\ Department of Pediatrics, Damascus University, Damascus, Syria}

Aim of the study: Studding serological inversion of hepatitis $A$ virus in children and knowing the percentage of asymptomatic hepatitis $A$.

Material and methods: This study was done in children's hospital in years 2007-2008. The number of children included in the study was 168 (88 male 
and 80 female). Children were between $1-13$ years of age and they were divided into 6 groups (24 months for each group). IgG and IgM for hepatitis $A$ were measured. Excluded of the study all patients with vomiting, diarrhea, jaundice, hepatic problems, infants have been vaccinated against hepatitis $A$ and anther sibling of the same family.Results: Medium age of patients was $7.3+/-2.1$ years. The percentage of lgG anti-hepatitis A positive was $47 \%$. Fig. (1) Type of epidemiology in the studyThe higher percentage of anti HAV-lgM positive was in 6-9 years of age (school age), while anti-HAV-lgG was mostly positive between 10-13 years of age.

Conclusions: Hepatitis A vaccine should be given to children in our country

\section{7}

\section{INFLIXIMAB IN THE TREATMENT OF PEDIATRIC IBD: A SINGLE CENTRE EXPERIENCE}

\author{
S. Accomando, C. Zicari, C. Albino, A. Liotta, \\ G. Corsello \\ University of Palermo, Palermo, Italy
}

Background and aims: The biological treatment of IBD acts on different stages of immunophysiopathological processes of the disease. We describe evolution and clinical response to Infliximab in paediatric patients affected by IBD diagnosed and followed at our centre.

Methods: In the last triennial period at our Department of Paediatrics in Palermo 53 infusions of Infliximab were administrated to our patients affected by severe forms of IBD. All patients but one were affected by several forms of CD. Only one child was affected by U.C. associated to pyoderma gangrenosum. The total number of infusions were administrated according the ACCENT 1 study at a dose of $5 \mathrm{mg} / \mathrm{Kg}$. All the infusions were preceded by e.v. administration of chlorpheniramine to avoid immunological reactions. The mean length of time of infusion was of 3 hours.

Results: Our experience on this biological drug was positive, in fact treated patients showed a good clinical response. None had adverse reactions. Only a 12 years old female patient with fistulising Crohn's disease, presented a flare of the disease one year after discontinuation of IFX. Some other patients, several months after the last infusion of Infliximab, were in remission state.
Conclusions: Infliximab has a great potential in improving the treatment also of pediatric IBD. However, its role in long-term therapy is not yet clear and it remains to be determined which is the long term tolerability of the drug and to examine if the effectiveness of the drug is reduced or not by the discontinuation of its administration.

\section{8}

\section{THE EFFECT OF CONSTIPATION TREATMENT ON VOIDING DYSFUNCTION IN CHILDREN}

\section{T. Shahraki}

Pediatric, Zahedan Medical Science of University, Zahedan, Iran

Background: Constipation and voiding dysfunction are common in children. The purpose of present study is to evaluate resolution of voiding dysfunction before and after treatment of constipation.

Material and Methods: 48 children (20month _11years) with evidence of voiding dysfunction and chronic constipation were evaluated in year 13871388. Patients treated for constipation for 3 months. Voiding dysfunctional score based on DVSS before and three months after treatment were compared. Girls with score $>6$ and boys with score of 9 were included.

Results: $35.4 \%$ and $65.4 \%$ of subjects were boys and girls respectively .Median score of voiding dysfunction revealed significant differences before and after treatment $(p=0.001)$.Comparison of scores in both sexes and ages were not significant. $(p=0.4,0.9$, respectively). After treatment, enuresis and encoprosis as well as frequency in defecation showed significant improvement among girls and boys. ( $p=0.003$ and 0.001 respectively).

Results: results of this study showed that treatment of constipation could be effective in improvement of voiding dysfunction in children. More studies recommend in this regard. 